Jan S. Hesthaven

Tim Warburton

\title{
Nodal Discontinuous Galerkin Methods
}

Algorithms, Analysis, and Applications 


\section{Contents}

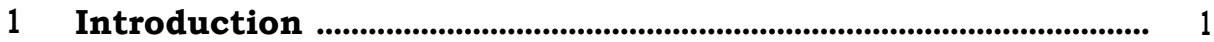

1.1 A brief account of history …………………………………….. 10

1.2 Summary of the chapters ............................................................... 13

1.3 On the use and abuse of the Matlab codes .................................. 16

1.4 Scope of text and audience ........................................................... 16

2 The key ideas .................................................................................... 19

2.1 Briefly on notation …………………………........................ 19

2.2 Basic elements of the schemes ...................................................... 20

2.2.1 The first schemes ............................................................. 20

2.2.2 An alternative viewpoint ……………………………...... 29

2.3 Toward more general formulations .................................................. 31

2.4 Interlude on linear hyperbolic problems …………….................. 34

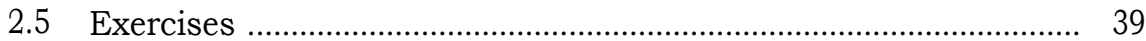

3 Making it work in one dimension ...................................................... 43

3.1 Legendre polynomials and nodal elements ................................. 43

3.2 Elementwise operations ............................................................... 51

3.3 Getting the grid together and computing the metric ……......... 56

3.4 Dealing with time .............................................................................. 63

3.5 Putting it all together ................................................................... 64

3.6 Maxwell's equations …………………………………………...... 67

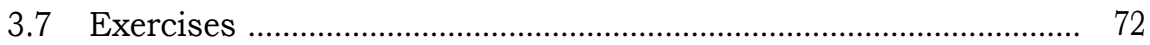

$4 \quad$ Insight through theory ……...................................................................... 75

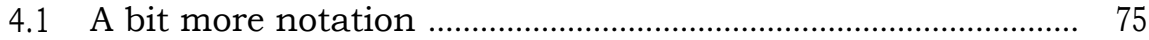

4.2 Briefly on convergence …………………………............................ $\quad 76$

4.3 Approximations by orthogonal polynomials and consistency .... $\quad 77$

4.4 Stability ……................................................................................ 83

4.5 Error estimates and error boundedness ………………………..... 85

4.6 Dispersive properties ....................................................................... 88 
4.7 Discrete stability and timestep choices …................................. 93

4.8 Taming the CFL condition ......................................................... 97

4.8.1 Improvements by mapping techniques ............................. 98

4.8.2 Filtering by co-volume grids ............................................ 102

4.8.3 Local timestepping ........................................................ 108

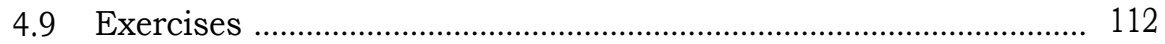

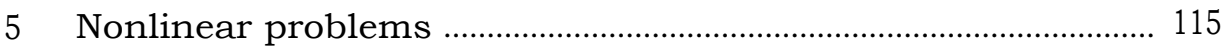

5.1 Conservation laws .......................................................................... 115

5.2 The basic schemes and their properties ....................................... 118

5.3 Aliasing, instabilities, and filter stabilization ............................. 123

5.4 Problems an nonconservative form ............................................. 134

5.5 Error estimates for nonlinear problems with smooth solutions 135

5.6 Problems with discontinuous solutions ...................................... 136

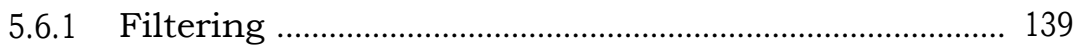

5.6.2 Limiting .............................................................................. 145

5.7 Strong stability-preserving Runge-Kutta methods ..................... 157

5.8 A few general results ................................................................. 160

5.9 The Euler equations of compressible gas dynamics .................... 161

5.10 Exercises .............................................................................. 165

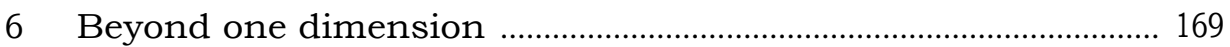

6.1 Modes and nodes in two dimensions ........................................... 171

6.2 Elementwise operations .......................................................... 183

6.3 Assembling the grid ......................................................................... 190

6.4 Timestepping and boundary conditions ..................................... 197

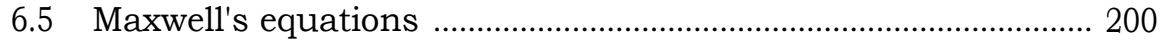

6.6 Compressible gas dynamics .......................................................... 206

6.6.1 Variational crimes, aliasing, filtering, and cubature integration ............................................................................. 210

6.6.2 Numerical fluxes revisited ................................................ 217

6.6.3 Limiters in two dimensions ............................................... 224

6.7 A few theoretical results ........................................................ 236

6.8 Exercises ................................................................................ 239

$7 \quad$ Higher-order equations ..................................................................... 243

7.1 Higher-order time-dependent problems ................................... 245

7.1.1 The heat equation ............................................................. 245

7.1.2 Extensions to mixed and higher-order problems ............ 255

7.2 Elliptic problems ...................................................................... 261

7.2.1 Two-dimensional Poisson and Helmholtz equations 275

7.2.2 A look at basic theoretical properties ............................. 287

7.3 Intermission of solving linear systems .......................................... 296

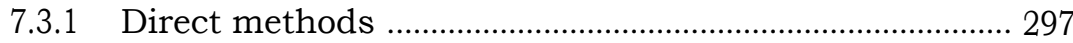

7.3.2 Iterative methods ................................................................ 299 
7.4 The incompressible Navier-Stokes equations .............................. 300

7.4.1 Temporal splitting scheme ................................................. 301

7.4.2 The spatial discretization ..................................................... 302

7.4.3 Benchmarks and validations .............................................. 308

7.5 The compressible Navier-Stokes equations .................................... 314

7.5.1 Integration-based gradient, divergence, and jump

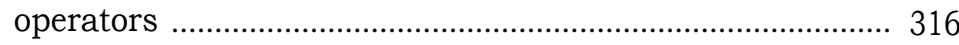

7.5.2 Solver for the compressible Navier-Stokes equations of gas dynamics ............................................................... 319

7.5.3 A few test cases ................................................................. 326

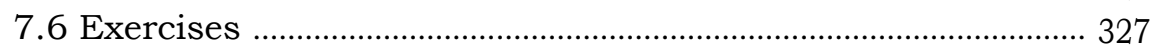

8 Spectral properties of discontinuous Galerkin operators ...... 331

8.1 The Laplace eigenvalue problem ...................................................... 334

8.1.1 Impact of the penalty parameter on the spectrum ........ 338

8.2 The Maxwell eigenvalue problem ……………………………...... 342

8.2.1 The two-dimensional eigenvalue problem ......................... 348

8.2.2 The three-dimensional eigenproblem ................................. 367

8.2.3 Consequences in the time domain .................................... 370

9 Curvilinear elements and nonconforming discretizations .... 373

9.1 Isoparametric curvilinear elements ................................................. 373

9.1.1 Forming the curvilinear element ......................................... 375

9.1.2 Building operators on curvilinear elements ...................... 378

9.1.3 Maxwell's equations on meshes with curvilinear

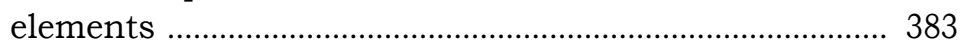

9.2 Nonconforming discretizations ………………………………...... 386

9.2.1 Nonconforming element refinement .................................. 387

9.2.2 Nonconforming order refinement ....................................... 396

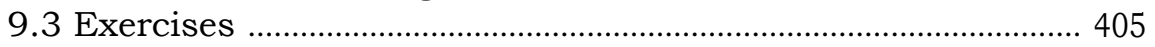

10 Into the third dimension ....................................................................... 407

10.1 Modes and nodes in three dimensions ………………………..... 409

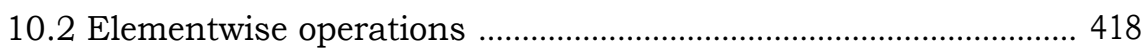

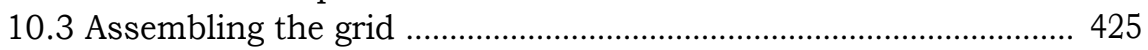

10.4 Briefly on timestepping .................................................................... 432

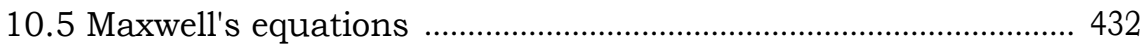

10.6 Three-dimensional Poisson equation ………………………….... 437

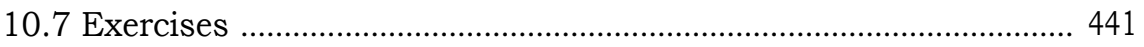

A Appendix A: Jacobi polynomials and beyond ............................. 445

A.1 Orthonormal polynomials beyond one dimension ....................... 448

B Appendix B: Briefly on grid generation ........................................... 451

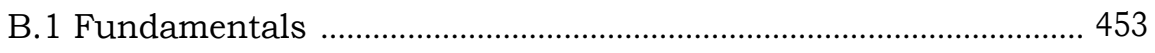

B.2 Creating boundary maps .............................................................. 457 
XIV Contents

C Appendix C: Software, variables, and helpful scripts .............. 461

C.1 List of important variables defined in the codes ........................... 461

C. 2 List of additional useful scripts .................................................... 465

References …..................................................................................................................................... 473

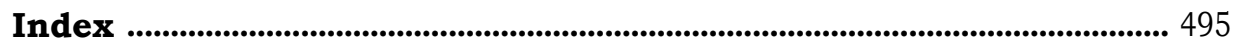

